\title{
Burkholderia cepacia Measurement
}

National Cancer Institute

\section{Source}

National Cancer Institute. Burkholderia cepacia Measurement. NCI Thesaurus. Code C147456.

The determination of the amount of the organism assigned to the Burkholderia cepacia species in a biological sample. 